\title{
A Novel Technique for Harvesting Hepatic Vein Blood and Time Course of Posthepatic Reactive Oxygen Species after Liver Transplantation in Rats
}

\section{Changku Jia ${ }^{1}$, Youke Chen ${ }^{1}$ and Honglei Liu ${ }^{2 *}$}

${ }^{1}$ Department of Hepatobiliary Surgery, Affiliated Hospital of Hainan Medical College, Haikou, P.R. China

${ }^{2}$ Office of Nosocomial Infection, Affiliated Hospital of Hainan Medical College, Haikou, P.R. China

\begin{abstract}
Objective: To introduce a method for harvesting pure hepatic vein (HV) blood and to determine time course and peak level of posthepatic (HV blood) reactive oxygen species (ROS) after liver transplantation.
\end{abstract}

Materials and methods: Blood was taken from HV and infrahepatic vena cava (IH-VC) for malondialdehyde (MDA) detection at different time points after transplantation.

Results: Pure posthepatic blood from HV was successfully obtained using the technique of IH-VC cannulation. This method guaranteed the harvesting of pure posthepatic blood from HV and pure peripheral blood from IH-VC. MDA concentration in $\mathrm{HV}$ elevated obviously with time and peaked at $1 \mathrm{~h}$ after transplantation. MDA concentration at $1 \mathrm{~h}$ was significantly higher than that at other time points except for 5 min. Furthermore, MDA concentrations in HV were significantly higher than that in IH-VC at $20 \mathrm{~min}, 30 \mathrm{~min}, 1 \mathrm{~h}(P<0.05)$ and slightly higher at all other time points. MDA concentration within $\mathrm{IH}-\mathrm{VC}$ peaked at $2 \mathrm{~h}$ after transplantation.

Conclusion: We introduced a novel technique for harvesting pure posthepatic blood from HV using the manipulation of IH-VC cannulation. We provided the first evidence that posthepatic ROS concentration elevated with time and peaked at $1 \mathrm{~h}$ after transplantation.

Keywords: Reactive oxygen species; Liver; Transplantation; Ischemia; Reperfusion; Hepatic vein

\section{Introduction}

Liver ischemia-reperfusion (I/R) occurs in a number of clinical syndromes, including liver transplantation [1]. Liver I/R is a major obstacle to liver transplantation [2,3]. It causes liver graft primary nonfunction, up to $10 \%$ of early liver transplant failures and higher incidence of acute and chronic rejection [3-5].

Multiple mechanisms are involved in the pathogenesis of liver transplant I/R in which reactive oxygen species (ROS) have been shown to play a central role in aggravating tissue injury of the transplant [6]. The generation of ROS during the reperfusion phase underlies the pathophysiology of this syndrome. Liver by itself is the main source of ROS and proinflammatory cytokines which responsible for the deleterious effects on the hepatocytes during hepatic I/R $[7,8]$. We hypothesized that in the early period of reperfusion ROS concentration in hepatic vein (HV) (posthepatic) differs from, may be higher than, that in infrahepatic vena cava (IH-VC) (peripheral). And in previous study, using rat warm I/R injury model, we demonstrated that posthepatic ROS concentration elevated gradually with time after reperfusion. And in the early period of reperfusion posthepatic ROS concentration is higher than that in infrahepatic vena cava (IH-VC) [9]. But little is known about time course and peak level of posthepatic (HV) reactive oxygen species after liver transplantation. In this study we introduced a method for harvesting pure posthepatic blood and evaluated the dynamic change of posthepatic ROS after liver transplantation in rats.

\section{Materials and Methods}

\section{Animals and reagents}

Male Sprague-Dawley rats, weighing 200-250 g, were supplied by the Shanghai Experimental Animal Center. Rats were housed under standard environmental conditions with a 12:12-h light-dark cycle. Before use in experiments, rats were fasted overnight with free access to water. All animals received human care in accordance with the Guidelines of the Animal Care Committee of Zhejiang Medical University. MDA and myeloperoxidase (MPO) assay kit (Jiancheng
Bioengineering Co.Ltd, Nanjing, China), Naphthol ASD chloroacetate esterase (Sigma Chemical Co., St. Louis, MO) and IL-6 ELISA assay kit (Quantikine MN, USA).

\section{Surgical procedures, experimental groups and sample harvesting}

The animals were anesthetized with intraperitoneal injection of $4 \%$ chloral hydrate. Orthotopic liver transplantation was performed by Kamada's two-cuff technique [10]. Pure posthepatic blood (HV) and IH-VC blood were taken at $5 \mathrm{~min}, 20 \mathrm{~min}, 30 \mathrm{~min}, 1 \mathrm{~h}, 2 \mathrm{~h}, 6 \mathrm{~h}$ after reperfusion, respectively ( $\mathrm{n}=7$ for each time point). Pure posthepatic blood and IH-VC blood were harvested by the technique of IH-VC cannulation and syringe puncture extraction, respectively. A 7\# silk suture was placed behind the IH-VC and just above the level of right adrenal vein. To obtain pure HV blood in different time points after reperfusion, an indwelling intravenous line (BD Vialon Biomaterial) was cannulated in the IH-VC with the catheter tip positioned between the liver and diaphragm. After cannulation a slipknot around the indwelling intravenous line was tied using the pre-placed 7\# silk suture to separate blood between both sides of the suture. Then superior hepatic vena cava was occluded at the level of diaphragm. Then pure HV blood was drawn-out through the catheter. Pure IH-VC blood was sampled by the method of syringe puncture under the ligated $7 \#$ silk suture. After the blood extraction, superior hepatic vena cava and IH-VC was orderly unblocked followed by the catheter withdrawn. A

*Corresponding author: Honglei Liu, Office of Nosocomial Infection, Affiliated Hospital of Hainan Medical College, 31 Longhua Road, Haikou, P.R. China, E-mail: 18976452639@126.com

Received February 16, 2012; Accepted March 27, 2012; Published April 02, 2012

Citation: Jia C, Chen Y, Liu H (2012) A Novel Technique for Harvesting Hepatic Vein Blood and Time Course of Posthepatic Reactive Oxygen Species after Liver Transplantation in Rats. J Transplant Technol Res S1:008. doi:10.4172/2161-0991. S1-008

Copyright: (c) 2012 Jia C, et al. This is an open-access article distributed under the terms of the Creative Commons Attribution License, which permits unrestricted use, distribution, and reproduction in any medium, provided the original author and source are credited. 
schematic representation for blood extraction is shown in Figure 1. Sera were separated for lipid peroxide detection.

\section{Lipid peroxide assay}

Blood MDA level, an indicator of oxidative injury, was determined by the thiobarbituric acid reaction. Sera were isolated then MDA was detected according to manufacturer's instructions.

\section{Statistical analysis}

These data are expressed as mean $\pm \mathrm{SD}$. Calculations were made using the SPSS 13.0 for windows computer software (SPSS Inc., Chicago, IL). Statistical comparisons were performed using one-way analysis of variance (ANOVA) and the LSD t-test for blood MDA levels. P-values less than 0.05 were considered statistically significant.

\section{Results}

\section{Success rate of sample harvesting}

Pure posthepatic blood from HV was successfully obtained using the technique of IH-VC cannulation. This method guaranteed the harvesting of pure posthepatic blood from $\mathrm{HV}$ and pure peripheral blood from IH-VC. And it was easy to obtain more than $2 \mathrm{ml}$ of $\mathrm{HV}$ blood in all animals (100\%), thus making the experiment successful.

\section{Time course and peak level of posthepatic MDA after transplantation}

Pure posthepatic blood and IH-VC blood were taken at $5 \mathrm{~min}, 20$ $\mathrm{min}, 30 \mathrm{~min}, 1 \mathrm{~h}, 2 \mathrm{~h}$ and $6 \mathrm{~h}$ after transplantation. Sera were isolated and MDA level in sera, an indicator of oxidative injury, was determined by the thiobarbituric acid reaction. MDA was detected according to manufacturer's instructions. Figure 2 represents the kinetic changes of MDA in all time points. In this study MDA concentration within HV peaked at $1 \mathrm{~h}$ after transplantation. And MDA concentration at $1 \mathrm{~h}$ was significantly higher than that at other time points except for $5 \mathrm{~min}$. Furthermore, MDA concentrations in HV were significantly higher than that in $\mathrm{IH}-\mathrm{VC}$ at $20 \mathrm{~min}, 30 \mathrm{~min}, 1 \mathrm{~h}(\mathrm{P}<0.05)$ and slightly higher

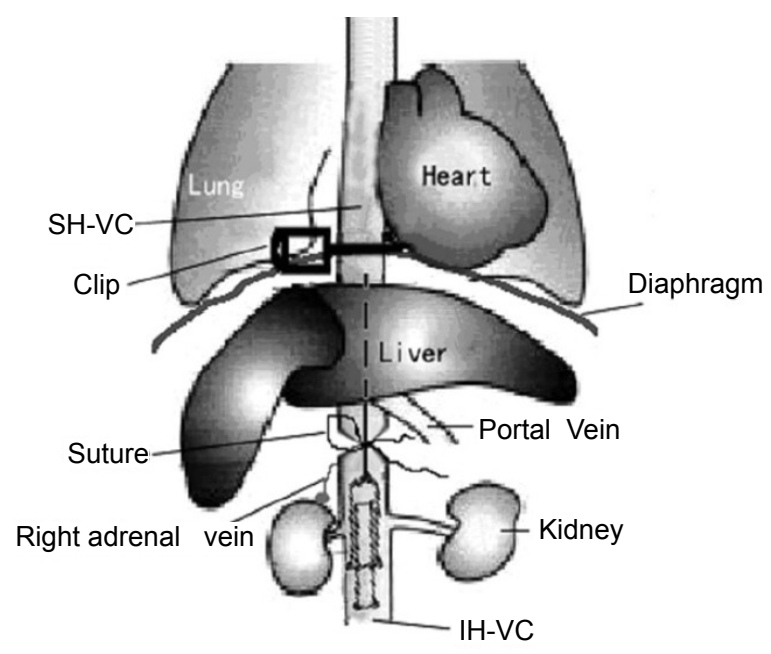

Figure 1: Schema of blood extraction from HV, IH-VC and sites of vascular occlusion. To obtain pure posthepatic blood, the vena cava was occluded between the liver and the right atrium and between the liver and right adrenal vein. Pure HV blood was drawn-out through the catheter. Meanwhile, pure $\mathrm{IH}$ $\mathrm{VC}$ blood was sampled by the method of syringe puncture under the ligated $7 \#$ silk suture.

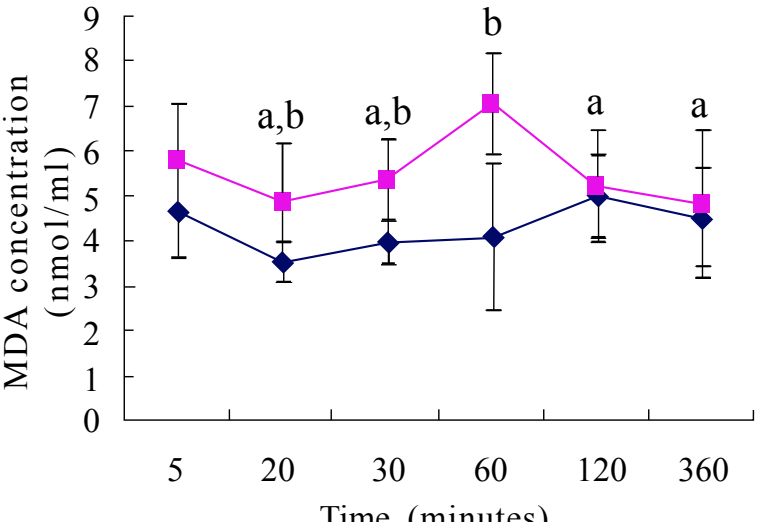

Figure 2: Time course and peak level of MDA after liver transplantation $\mathrm{HV}$ represents the group that blood was taken from hepatic vein. IH-VC represents the group that blood was taken from IH-VC. ${ }^{a} P<0.05$ vs. 60 min within HV. ${ }^{\mathrm{b}} P<0.05$ vs. IH-VC. ${ }^{\mathrm{a}} P$ : HV. ${ }^{\mathrm{b}} P$ : IH-VC.

at all other time points. MDA concentration within IH-VC peaked at $2 \mathrm{~h}$ after transplantation.

\section{Discussion}

Some measurements of pure blood sample from the hepatic vein without backflow contamination of blood from the caval vein will be needed in some experiments. Backflow from the right heart or caval vein into the hepatic vein has been well recognized clinically for a number of years and can be easily visualized by following the flow of dye injected into a hepatic vein [11]. This is an obstacle to analyze liver metabolism regarding blood from the hepatic vein without backflow contamination. In this study, we introduced a novel technique for harvesting pure posthepatic blood from hepatic vein using the manipulation of IH-VC cannulation. This method guaranteed the harvesting of pure posthepatic blood from HV without backflow contamination and pure peripheral blood from IH-VC, and it was easy to obtain enough volume of HV blood in all animals (100\%), thus making the experiment smoothly completed.

Regarding the time course and peak production of posthepatic ROS, our results provided evidence for the role of liver transplant in generating ROS after reperfusion. In this study, pure HV and IHVC blood were taken at multiple time points to determine the peak production of posthepatic ROS and the differences between them after transplantation. We found that serum MDA concentration in $\mathrm{HV}$ increased and peaked at $1 \mathrm{~h}$ after transplantation, and the MDA concentrations in $\mathrm{HV}$ were significantly higher than that in $\mathrm{IH}-\mathrm{VC}$ at 20 min, $30 \mathrm{~min}, 1 \mathrm{~h}(P<0.05)$. The phenomenon that MDA concentration increased slowly in the early stage after transplantation is due to its incomplete reflow and injured microcirculation including endothelial cell swelling [12], vasoconstriction [13], leucocyte entrapment [14,15] and possibly intravascular haemoconcentration [16]. This process prolongs the period of hypoxia, with areas of the liver remaining ischemic after early period of transplantation. In the later stage, neutrophils infiltrate into the liver increased with time, so companied by increased ROS.

Based on this founding, efforts might be made to alleviate hepatic cold I/R injury in different time points. In liver transplantation, for example, means might be used to scavenge ROS in the phase of peak production in posthepatic blood (HV), in the phase of peak production in peripheral blood (IH-VC) or in both phases after transplantation, 
Citation: Jia C, Chen Y, Liu H (2012) A Novel Technique for Harvesting Hepatic Vein Blood and Time Course of Posthepatic Reactive Oxygen Species after Liver Transplantation in Rats. J Transplant Technol Res S1:008. doi:10.4172/2161-0991.S1-008

respectively. It might improve the overall success of liver transplantation and should be further investigated at least in animal models.

\section{Funding}

This research was supported by the National Natural Science Foundation of China (81160037)

\section{References}

1. Huguet C, Gavelli A, Bona S (1994) Hepatic resection with ischemia of the liver exceeding one hour. J Am Coll Surg 178: 454-458.

2. Rosen HR, Martin P, Goss J, Donovan J, Melinek J, et al. (1998) Significance of early aminotransferase elevation after liver transplantation. Transplantation 65: 68-72.

3. Banga NR, Homer-Vanniasinkam S, Graham A, Al-Mukhtar A, White SA, et al. (2005) Ischaemic preconditioning in transplantation and major resection of the liver. Br J Surg 92: 528-538.

4. Henderson JM (1999) Liver transplantation and rejection: an overview. Hepatogastroenterology 46: 1482-1484

5. Farmer DG, Kaldas F, Anselmo D, Katori M, Shen XD, et al. (2008) Tezosentan, a novel endothelin receptor antagonist, markedly reduces rat hepatic ischemia and reperfusion injury in three different models. Liver Transpl 14: 1737-1744.

6. Zhang W, Wang M, Xie HY, Zhou L, Meng XQ, et al. (2007) Role of reactive oxygen species in mediating hepatic ischemia-reperfusion injury and its therapeutic applications in liver transplantation. Transplant Proc 39: 1332-1337.

7. Urata K, Brault A, Rocheleau B, Huet PM (2000) Role of Kupffer cells in the survival after rat liver transplantation with long portal vein clamping times. Transpl Int 13: 420-427.
8. Shibuya H, Ohkohchi N, Seya K, Satomi S (1997) Kupffer cells generate superoxide anions and modulate reperfusion injury in rat livers after cold preservation. Hepatology 25: 356-360

9. Jia C, Wang W, Zhu Y, Zheng S (2008) Suprahepatic vena cava manipulative bleeding alleviates hepatic ischemia-reperfusion injury in rats. Dig Liver Dis 40: $285-292$

10. Kamada N, Calne RY (1983) A surgical experience with five hundred thirty liver transplants in the rat. Surgery 93: 64-69.

11. Irtun O, Martini WZ, Ozkan O, Wolfe RR (2001) Caval backflow: a potentia problem during blood sampling from the hepatic vein. Metabolism 50: 189-193.

12. Vollmar B, Glasz J, Leiderer R, Post S, Menger MD (1994) Hepatic microcirculatory perfusion failure is a determinant of liver dysfunction in warm ischemia-reperfusion. Am J Pathol 145: 1421-1431.

13. Marzi I, Takei Y, Rücker M, Kawano S, Fusamoto H, et al. (1994) Endothelin-1 is involved in hepatic sinusoidal vasoconstriction after ischemia and reperfusion. Transpl Int 7: S503-S506.

14. Fondevila C, Busuttil RW, Kupiec-Weglinski JW (2003) Hepatic ischemia/ reperfusion injury--a fresh look. Exp Mol Pathol 74: 86-93.

15. Yadav SS, Howell DN, Gao W, Steeber DA, Harland RC, et al. (1998) L-selectin and ICAM-1 mediate reperfusion injury and neutrophil adhesion in the warm ischemic mouse liver. Am J Physiol 275: G1341-G1352.

16. Menger MD, Sack FU, Barker JH, Feifel G, Messmer K (1988) Quantitative analysis of microcirculatory disorders after prolonged ischemia in skeletal muscle. Therapeutic effects of prophylactic isovolemic hemodilution. Res Exp Med (Berl) 188: 151-165. 